\title{
Factores que influyen en la satisfacción del paciente de diálisis con enfermería
}

\author{
María Sanz Turrado, Luis Garrido Pérez, Carmen Caro Domínguez \\ Facultad de Medicina y Enfermería. Universidad de Córdoba. España
}

\section{Resumen}

Introducción: La satisfacción del paciente con los cuidados enfermeros se ha convertido en un determinante clave de la calidad de la atención sanitaria. Además, esto es especialmente relevante, sobre todo en pacientes crónicos, como es el caso del paciente con enfermedad renal crónica avanzada en hemodiálisis.

Objetivo: Analizar los factores que intervienen en la satisfacción de los pacientes en hemodiálisis respecto al personal de enfermería.

Metodología: Se ha llevado a cabo un estudio cualitativo, de tipo fenomenológico, mediante grupo focal de 7 pacientes en hemodiálisis de la Unidad de Gestión Clínica de Nefrología de Córdoba. Los sujetos fueron seleccionados mediante muestreo intencionado, tras su consentimiento informado.

Resultados: Se han categorizado los resultados según las dimensiones de la calidad percibida. Respecto a la accesibilidad creen que son bien atendidos, pero pierden mucho tiempo en la unidad de diálisis. En cuanto a la fiabilidad, confían en las enfermeras con destreza para pinchar la fístula y desconfían de las enfermeras nuevas. Respecto a la comunicación, resaltan la buena relación existente entre ellos y el personal de enfermería. El trato es percibido como un punto fuerte, agradable y humano, en general. De forma generalizada destacan en las enfermeras un alto grado de competencia profesional, ligada al grado de fiabilidad que tengan con la enfermera. En general, califican el

Correspondencia:

María Sanz Turrado

$\mathrm{C} /$ Cronista Salcedo Hierro $\mathrm{n}^{\circ} 3$, portal 4, $5^{\circ} \mathrm{D}$

14001 Córdoba. España

E-mail: n12satum@uco.es servicio asistencial que reciben de muy bueno e incluso excelente.

Conclusiones: Este grupo de pacientes en hemodiálisis destaca la relación que se crea entre ellos y enfermería. Tienen un alto grado de confianza en el personal de enfermería, basado en su destreza para pinchar la fístula arteriovenosa y en su fiabilidad profesional.

PALABRAS CLAVE

- SATISFACCIÓN DEL PACIENTE

- HEMODIÁLISIS

- GRUPO FOCAL

Factors influencing the degree of satisfaction of the hemodialysis patient with nursing

\section{Abstract}

Introduction: Patient satisfaction with nursing care has become a key determinant of the quality of health care. In addition, this is especially relevant, especially in chronic patients, such as patients with advanced chronic kidney disease on hemodialysis.

Objective: To analyze the factors involved in the satisfaction of patients on hemodialysis regarding nursing staff.

Methodology: A qualitative, phenomenological study was carried out by focal group of 7 patients on hemodialysis of the Clinical Management Unit of Nephrology of Córdoba. Subjects were selected by intentional sampling, after their informed consent.

Results: The results were categorized per the dimensions of perceived quality. Regarding accessibility, 
they believe that they are well taken care of, but they lose a lot of time in the dialysis unit. As for reliability, they rely on nurses who have the skill to puncture the fistula, and are wary of new nurses. Regarding communication, they highlight the good relationship between them and the nursing staff. The treatment is perceived as a strong point, pleasant and human in general. In general, nurses have a high degree of professional competence, linked to the degree of reliability they have with the nurse. In general, they rate the service they receive from very good and even excellent.

Conclusions: This group of hemodialysis patients highlights the relationship between them and nursing. They have a high degree of confidence in the nursing staff based on their ability to puncture the arteriovenous fistula and in their professional reliability.

\section{KEYWORDS}

- PATIENT SATISFACTION

- HEMODIALYSIS

- FOCAL GROUP

\section{Introducción}

En el actual sistema sanitario, el ciudadano se ha convertido en centro y motor del mismo. Las necesidades del paciente son la base sobre la que se articulan las prestaciones asistenciales y la organización de los servicios sanitarios ${ }^{1}$. De hecho, la satisfacción del usuario es considerada en la actualidad como parte de los resultados de la asistencia sanitaria ${ }^{2}$. La valoración que el paciente realiza de la atención recibida es un indicador importante del funcionamiento de un servicio en particular y del sistema sanitario en general ${ }^{3}$.

El concepto de satisfacción se basa en la diferencia entre las expectativas del paciente y la percepción de los servicios que ha recibido; es decir, la percepción subjetiva, acompañada de la expectativa previa ${ }^{4}$. Es un concepto complejo que está relacionado con una gran variedad de factores, como son el estilo de vida, las experiencias previas, las expectativas de futuro y los valores del individuo y de la sociedad 5 . La satisfacción de las necesidades y expectativas del paciente se considera una medida de eficacia, confort, pertinencia y control del funcionamiento del sistema sanitario $0^{6,7}$. Por tanto, no van a tener las mismas expectativas y percepciones de la asistencia sanitaria un paciente crónico y un usuario ocasional (in- tervención quirúrgica, proceso agudo, etc) ${ }^{8}$. Los pacientes crónicos son usuarios regulares del sistema sanitario, por lo que su percepción va a estar influenciada por el continuo contacto con el mismo?.

Por otro lado, los cuidados de enfermería son una parte fundamental del manejo clínico del paciente y los estudios ponen de manifiesto que la satisfacción con estos cuidados es el aspecto que mejor predice la satisfacción con la hospitalización en general|10-14. En consecuencia, la satisfacción del paciente con los cuidados enfermeros se ha convertido en un determinante clave de la calidad de la atención sanitaria ${ }^{15,16}$.

Para conocer la opinión de los usuarios respecto al personal de enfermería, la mayoría de los estudios están basados en las encuestas de satisfacción ${ }^{17-19}$. Los instrumentos de medida más utilizados para evaluar la satisfacción del usuario son estructurados y tienen la ventaja de poderse analizar estadísticamente, pero en ocasiones, pueden no explorar en profundidad percepciones que no están incluidas en los cuestionarios ${ }^{20}$.

Esto puede ser especialmente relevante en pacientes crónicos como el paciente con Enfermedad Renal Crónica Avanzada (ERCA) en tratamiento con hemodiálisis (HD) periódicas. Los estudios que analizan el grado de satisfacción del paciente en HD han utilizado como instrumento de medida el Cuestionario SERVQHOS (cuestionario para evaluar la calidad percibida de la atención hospitalaria), adaptado al paciente en diálisis ${ }^{21}$.

En general, estos estudios reflejan que se valoran en mayor medida aspectos subjetivos de la asistencia sanitaria y destacan que la comunicación entre profesionales y pacientes juega un papel fundamental en la satisfacción del usuario, aunque muestran una homogeneidad bastante común en sus resultados ${ }^{22,23}$; por lo que es difícil establecer qué aspectos concretos de la aportación de enfermería al proceso asistencial son los más valorados.

En este sentido, cuando el objetivo del estudio es analizar el punto de vista del usuario implicado en el sistema sanitario, hay cierta información de difícil acceso para el investigador cuantitativo. En estos casos, los métodos cualitativos resultan especialmente adecuados para la comprensión en profundidad este fenómeno que se desea investigar o evaluar ${ }^{24}$.

Por este motivo nos planteamos el siguiente estudio, con el objetivo de analizar los factores que intervienen en la satisfacción de los pacientes en HD respecto al personal de enfermería. 


\section{Material y Método}

\section{Diseño}

Se ha llevado a cabo un estudio cualitativo utilizando para ello un enfoque fenomenológico.

\section{Sujetos de estudio}

La muestra estuvo formada por 7 pacientes. Se seleccionó de forma no probabilística intencional, de un grupo de pacientes con ERCA en HD periódicas, de la Unidad Hospitalaria de Diálisis de la Unidad de Gestión Clínica (UGC) de Nefrología del Hospital Universitario Reina Sofía de Córdoba. La muestra fue intencional porque se buscó a pacientes con capacidad para interaccionar con la entrevistadora y con un perfil relativo de "objetividad". Esta selección fue realizada por el responsable de enfermería de esta Unidad.

\section{Técnica de recogida de información}

La técnica de recogida de información ha sido el Grupo Focal. La reunión tuvo una duración de una hora y se llevó a cabo después de la sesión de diálisis, para evitar desplazamientos y pérdidas de tiempo añadidos para los pacientes. Esta sesión se realizó fuera de la unidad de diálisis y fue conducida por una moderadora en colaboración con una observadora que se encargaba de tomar notas y grabar. Después se desarrolló una reunión entre ambas para intercambiar impresiones. La información obtenida fue traspasada a formato digital para ser posteriormente analizada.

\section{Análisis de los datos}

El análisis de la información se lleva a cabo con la elaboración de un informe donde se recogen las impresiones obtenidas en la sesión, incluyendo fragmentos de las trascripciones, cuyo contenido refuerza las opiniones emitidas. El análisis de los datos se ha realizado sobre las dimensiones de la calidad percibida de accesibilidad, trato e información del modelo SERVQUAL de Parasuraman ${ }^{21}$, identificándose las fortalezas y debilidades que, según los asistentes, presenta la asistencia realizada por el personal de enfermería en la Unidad de Diálisis, recogidas a través de las opiniones que más se repiten y en las que existe un alto grado de consenso.

\section{Consideraciones éticas}

El estudio se realizó después de que los pacientes aceptaran voluntariamente participar en el mismo, tras ser informados verbalmente y dar su consentimiento para participar en el estudio; y tras obtener el Informe FAVORABLE para la realización del mismo por parte del Comité Provincial de Ética de la Investigación de Córdoba.
Todos los datos fueron tratados de acuerdo a la Ley Orgánica de Datos de Carácter Personal 15/1999, garantizándose en todo momento la confidencialidad de los mismos y el anonimato de los pacientes.

\section{Resultados}

De forma general los asistentes se muestran muy satisfechos con la atención recibida por parte de las enfermeras de la Unidad de Diálisis, destacando la seguridad que sienten cuando son atendidos en esta Unidad y que al comparar con otros centros de Diálisis, todos los asistentes sin excepción afirman que solo quieren ser atendidos aquí, "a la pregunta, ¿se cambiarían de centro si pudiesen? Todos contestan con un NO rotundo".

Asimismo, destaca de forma general, la positiva percepción del buen trato que reciben de las enfermeras, expresado en términos de "humano", "cercano" y "amable", apuntando que el interés que éstas profesionales demuestran hacia ellos, va más allá del interés propio por su situación de salud, y expresan que, de forma general, todas las enfermeras se interesan por los temas que a los pacientes les preocupan. Consideran que dentro de lo "malo" de la enfermedad, están muy bien gracias al trato que reciben y al vínculo tan cercano que se crea entre pacientes-profesionales.

En relación a qué cosas podrían mejorarse, destacan aspectos estructurales como ampliación de la unidad para dotarla de más puestos de diálisis y por tanto reducción de los tiempos de espera, y mejoras en la comida que se les facilita durante su estancia en la Unidad.

Siguiendo el modelo SERVQUAL, se categorizaron los resultados según las dimensiones de la calidad percibida.

\section{Accesibilidad}

Lo mejor respecto a esta variable, es que sienten que son atendidos, escuchados e informados siempre que lo necesitan.

Lo peor valorado, es que teniendo en cuenta que son personas que pasan alrededor de cuatro horas, tres días a la semana, el tiempo que pasan en diálisis es muy relevante para ellos. Por lo tanto, hacen hincapié en los tiempos de espera para el inicio del tratamiento. Son conscientes de que hay que priorizar con aquellas personas que más tiempo de diálisis necesitan. Pero comentan que se podría agilizar el proceso ampliando la capacidad de la Unidad. 
"Si porque nos tienen a veces mucho tiempo en espera...Porque claro, Ilegas de la calle, Ilegas de tu casa y tú lo que quieres es que te conecten y claro tienes que esperar un tiempo razonable y lo más razonable es que pinchen antes al que más horas tiene y el que menos horas tiene pues..."(Paciente 1)

"...en su momento se habló aquí que la unidad de diálisis iba a ser más grande y yo pienso que hay poca capacidad. Porque a veces vienen personas que vienen malas y tienen que esperar a que alguno termine para poderlo poner" (Paciente 2)

\section{Fiabilidad}

Dentro de las cualidades que debe tener una enfermera para ellos, una de las más valoradas es la destreza pinchando, "una enfermera es buena si pincha bien", es decir, si consigue canalizar la fístula arteriovenosa a la primera.

Por lo tanto, depositan su confianza en aquellos profesionales que ya conocen y que anteriormente han conseguido realizarle su tratamiento de diálisis sin incidencias relevantes.

Relacionan la eficacia de la diálisis con el profesional que les ha atendido.

"Incluso hay personas que te pinchan bien y hay otros días que no te pinchan bien, pero pasa eso, te da más confianza el que sabes que va a hacerlo bien que el que dices: uf, con esta me ha dado problemas, a ver cómo vamos"(Paciente 1$)$

"Ya nada más que con pensar en quién viene el lunes por ejemplo, sabemos que esa semana ya para otra queda"(Paciente 3)

Máxima confianza en el supervisor de la Unidad. Esta figura representa para ellos la salvación cuando el resto del personal de enfermería no consigue canalizar la fístula.

"Estamos deseando de ver al supervisor asomar por la puerta"(Paciente 1)

"A él, sí" (Paciente 4)

"Da tranquilidad"(Paciente 6)

Como aspecto negativo, la existencia de un sentimiento general de desconfianza cuando llega personal nuevo a la unidad, concretamente en las temporadas de vacaciones.
"Estamos temiendo las vacaciones" (Paciente 4)

"Ahora cuando llega el verano ya es mortal. Porque eso ya es preguntar a la semana, porque como entra gente nueva porque ellas tienen vacaciones, pero debe quedarse gente de las que saben pinchar o de los que Ileven más tiempo pinchando, aunque los otros ya saben algo" (Paciente 1)

Es curiosa la diversidad de opiniones que existen en función de las características del acceso venoso que tiene cada uno:

- Paciente que porta catéter venoso: todas las enfermeras son excelentes y le tratan genial, no tiene queja. Apunta que "le puede atender cualquiera".

"Yo no puedo hablar de si pinchan bien o pinchan mal. Porque a mí no me pinchan [...] A mí me vale cualquiera para "engancharme" porque por lo demás no me pinchan"(Paciente 7)

- Paciente con fístula arteriovenosa sin complicaciones:

"Hay algunas que pinchan mejor que otras pero siempre se pueden equivocar, luego hay nuevas que también lo hacen muy bien y tienen que aprender [...] Yo eso no lo pregunto, quién va a tocar la semana que viene" (Paciente 6)

- Pacientes con fístula arteriovenosa complicada: sé que enfermeras me pinchan bien y temo cuando veo a alguna nueva.

"Claro porque a ti te pillan y te ponen (refiriéndose al compañero cuya fístula no presenta complicaciones). Pero los que no, ya sales más tarde, te duele más el brazo. Eso también te hace que tú digas uf como voy... incluso hace que salgas de la diálisis más tarde que otros días... y es la pescadilla que se va comiendo la cola. Si falla en el pinchazo y luego si va bien la diálisis bien, pero si empieza a pitar porque suba la presión, porque baje... ya eso es más tiempo y más problemas que tienes que tocar" (Paciente 1)

\section{Comunicación/ información}

Todos conocen sin excepción el nombre de los profesionales de enfermería que los atienden y afirman que a su llegada a la Unidad la enfermera que le atendió se presentó. 
Perciben que los enfermeros sienten interés por ellos, ya no solo en el ámbito físico o de la enfermedad, sino en el ámbito psicosocial.

Notan que las enfermeras se vuelcan en los problemas que puedan tener e intentan solucionarlos dentro de sus posibilidades.

"Claro, cada uno somos de nuestro padre y de nuestra madre. Hay algunas que son, que se meten en lo que a ti te está pasando...es que lo viven"(Paciente 2)

"No, que digo que como lo viven ellas mismas los problemas que uno tiene. Hay unas que lo viven más de cerca, otras un poquito más distanciado, pero yo las veo muy humanas"(Paciente 5)

"Hablan contigo, te dan su opinión de las cosas. Y vamos que se... no sé cómo decirlo. Pero que se mezclan con uno" (Paciente 6)

"Nuestra segunda casa, entonces que mejor que tener esas personas y preguntarle tú y le preguntas y le ves la cara y te das cuenta y te cuentan y tú les cuentas y yo pienso que podías hacer esto... vamos problemas, soluciones. Que para ti parece un mundo y de pronto tu empiezas a hablar y dices, si esto es una tontería..." (Paciente 2)

"Hay mucho roce, se cuentan cosas unos a otros, nos contamos e intentamos solucionar los problemas. Se habla mucho, si" (Paciente 3)

\section{Cortesía / trato}

El trato es la segunda cualidad del personal de enfermería más valorada por los pacientes.

Refieren un alto nivel de satisfacción general con el trato recibido en esta Unidad (la excepción es un caso muy concreto de un conflicto entre una paciente y una enfermera, pero lo reconocen como algo puntual y excepcional).

"Yo también digo lo mismo, que se están portando muy bien, por lo menos conmigo, con el resto no lo sé, pero conmigo se están portando muy bien"(Paciente 7)

"Si yo estoy contento con la forma en que me tratan y de la forma que me tratan. [...] Se sabe que cualquier día por ahí venimos con los cables cruzados, que no es lógico, porque no tiene nada que ver a como yo pueda estar con ellos y no tratarlas como deben [...] entonces también debes confrontar cualquier problema"(Paciente 5)
"Son todas muy cariñosas, muy amables, que da gusto tratar con ellas" (Paciente 6)

Perciben de forma generalizada un trato agradable y muy humano, reconociendo que se establecen vínculos con los profesionales que excede el trato profesional, llegando a tener un trato muy familiar y entablándose un clima de confianza, llegando a nombrarlos (a los profesionales) como su segunda familia.

"Yo el año pasado mismo, estuve más en el otro lado que aquí porque en $48 \mathrm{~h}$ me pusieron tres trasplantes, dos hígados y un riñón. Y yo notaba que tenían un sentimiento como si fuera un ser querido suyo. Pero no solo conmigo, sino con mi madre..."(Paciente i)

"Yo he tenido un problema en mi casa y ellas han estado ahí. Tanto las enfermeras como las auxiliares" (Paciente 4)

"Conmigo también, siempre preguntando con mi marido y por mí. No hay un día que no me pregunten" (Paciente 3)

"Son familiares, son familiares. Han vivido todo con nosotros" (Paciente 6)

\section{Competencia}

De forma generalizada destacan en las enfermeras un alto grado de competencia profesional, indicando que muestran un alto nivel de conocimientos en el desempeño de su trabajo.

Bajo el criterio de los pacientes de diálisis, la percepción de la competencia profesional va ligada al grado de fiabilidad que tengan con el enfermero o enfermera.

En general, califican el servicio asistencial que reciben de muy bueno e incluso excelente.

"Yo no tengo nada malo que decir, contenta con todos. Por mí son todas excelentes" (Paciente 3)

"Nada yo digo lo mismo, que yo de aquí no tengo queja, son todas muy buenas" (Paciente 5)

Están satisfechos con la organización del personal, ya que los más veteranos en el servicio atienden siempre el módulo donde más complicaciones con las fístulas suele haber.

Sin embargo, perciben una sobrecarga de trabajo en el equipo de enfermería, que influye en la asistencia clínica. 
"Para mí son todas excelentes, y todos los que están, pero también veo muchas veces que no pueden ser tan excelentes porque falta muchas veces personal" ( $\mathrm{Pa}$ ciente 1)

"Entonces eso es falta de personal, ¿y quiénes son los que lo pagamos? Nosotros porque no hay bastante gente" (Paciente 2)

"Pero llega un momento en el que ellas mismas se frustran porque ven que no dan abasto. Entonces que ocurre, que se pueden cabrear y a lo mejor, no es mi caso, pero pueden darte una mala contestación" ( $\mathrm{Pa}$ ciente 5)

\section{Seguridad}

Los pacientes de diálisis relacionan la fiabilidad en el personal con la seguridad física. Están seguros con aquellas enfermeras que conocen y ya le han tratado.

Remarcar también que una vez que tienen confianza en un enfermero, aceptan que este "les pinche mal" pues asumen que nadie es infalible. Su argumento es: "si él me ha pinchado mal hoy, imagina esa otra donde me pincharía"

"Al supervisor o a algunos como él que también hay. (EI supervisor) hay veces que te pega el pinchazo y piensas que es Dios para ti, en ese momento y dices: uy! Que bien nos toca. $Y$ si ese día te pincha mal, y dices, bueno, si él me ha pinchado mal a ver esa de enfrente que no me ha pinchado nunca imagínate donde me pincha. $Y$ es que es asi" (Paciente 2)

Manifiestan incertidumbre y miedo cuando les trasladan a otros centros de diálisis (ya sea por vacaciones propias o por reformas en sus centros). El motivo: no conocen cómo son las enfermeras del otro centro ni como les van a asistir.

Remarcar también la importancia en la percepción de la seguridad del paciente, el papel de la enfermera de diálisis cuando Ilegan nuevos a la Unidad:

"Pero al principio llegabas, tu llegas allí nuevo, con mucho miedo, te cogen la mano (las enfermeras), te dan mucha confianza y eso me llena a mí también [...] te has sentido protegido" (Paciente 6)

\section{Credibilidad}

Todos manifiestan u alto nivel de confianza en esta Unidad de diálisis. Sentimiento general de confianza en el equipo que les atiende.
"Si hay confianza, con varias. Yo tengo confianza con muchas. Y a mí me tratan, la mayoría me tratan bien, ¿Por qué voy a decir lo contrario?"(Paciente 7)

Manifiestan incertidumbre y miedo cuando les trasladan a otros centros de diálisis (ya sea por vacaciones propias o por reformas en sus centros). El motivo: no conocen cómo son las enfermeras del otro centro ni como les van a asistir.

"De hecho cuando cogemos vacaciones, porque también cogemos nosotros vacaciones, pues vas con la incertidumbre de a dónde vas y a ver cómo te van a tratar" (Paciente 1)

\section{Tangibilidad}

Consideran que el espacio en el que se ubica la Unidad de Diálisis está anticuado. Verían aceptable un cambio de sitio/sala.

\section{"Deberían cambiarnos de sitio"(Paciente 1)}

"Está muy anticuado ya, el sitio"(Paciente 2)

\section{Aceptabilidad/participación del paciente en su proceso}

La gran parte de los pacientes de diálisis son muy conscientes de su estado de salud y tienen conocimientos acerca de su enfermedad y tratamiento. Por ello, dentro de las complicaciones de la enfermedad, agradecen ser tratados en esta Unidad.

"Yo en mi caso los Reyes Magos vinieron el día 14 de octubre que fue cuando yo entre aquí. Eso es lo que yo me pedí a los reyes. Hace dos años yo estaba fatal y ahora sin embargo estoy bien, estoy estupendamente, como si no estuviera enfermo"(Paciente 6)

"Dentro de lo horroroso (de la enfermedad) se está bastante, bastante bien"

\section{Otros aspectos que destacan}

Todos destacan la falta de personal de enfermería en todas las Unidades de diálisis.

Perciben diferencias significativas en cuanto a trato y a medios entre los servicios de diálisis Públicos y los Concertados, teniendo una opinión muy negativa de estos últimos.

Quejas respecto a la calidad de la comida que se les facilita durante las sesiones de diálisis y al exceso de control que hay sobre ella. 


\section{Discusión}

La metodología cualitativa facilita el conocimiento en profundidad de las vivencias y necesidades de los pacientes. Los usuarios de diálisis pasan más de doce horas semanales en la Unidad, llegando a afirmar que es su "segunda casa". Por ello, es importante conocer la opinión del paciente respecto a los profesionales y el entorno en el que son tratados.

Cuando se analiza la calidad percibida por los usuarios respecto a enfermería, la satisfacción a nivel general es alta, lo cual coincide con la mayoría de los estudios. En todos, igual que en nuestros resultados se destaca que gran parte de los pacientes conocen el nombre de su enfermera, que lo peor valorado son los tiempos de espera, y los atributos más valorados son: amabilidad y disposición del personal para ayudar, preparación técnica del personal, interés del profesional para solucionar los problemas, la confianza y seguridad que el personal transmite y el trato personalizado 17-19,24-27,28.

Esto refuerza la idea de que los pacientes de diálisis tienen como principal indicador de satisfacción el trato recibido, siendo éste es un aspecto sobre el que se puede actuar directamente.

Otra dimensión de gran importancia es la fiabilidad. Se fían más de aquellas enfermeras que ya conocen, por el vínculo que se ha creado entre ellos, sintiéndose más seguros y satisfechos cuando les atiende "su enferme$\mathrm{ra}^{\prime \prime}$. Esto ha sido destacado también por otros autores: "la satisfacción del usuario puede depender más de las cualidades del personal sanitario y de la interacción entre ambos, o de la relación de ayuda que se establece entre enfermera-paciente" ${ }^{\prime \prime 19}$. Para algunos pacientes, la fiabilidad va ligada a la destreza para puncionar la fístula arteriovenosa. En efecto es la competencia profesional más destacada para ellos, porque asumen que cuando se les ha "pinchado" bien la diálisis transcurrirá sin incidencias.

Otro aspecto a destacar, como también se indica en los resultados, es que la fiabilidad va muy unida a la seguridad. La percepción de seguridad no sólo está relacionada con aspectos exteriores como el mantenimiento adecuado de los monitores, sino también con factores como la confianza en la competencia profesional, la disponibilidad de una información exhaustiva, la facilidad en el acceso a los profesionales para consultar dudas y la posibilidad de participar en los procesos de toma de decisión, tal como han destacado otros autores ${ }^{29}$.
Se ha comentado que la falta de personal provoca la frustración de las enfermeras y que, a veces, influye en la calidad de su trabajo. También existen algunos trabajos que reivindican una asociación entre la satisfacción de los profesionales sanitarios y la de sus pacientes, aspecto a tener en cuenta y ante el cual se podrían diseñar intervenciones en la gestión clínica con el fin de mejorar la satisfacción de ambos ${ }^{30,31}$.

En este estudio no se han tenido en cuenta las características sociodemográficas, ya que se buscaba la opinión general de los pacientes de la Unidad. Sin embargo, se ha demostrado en varios artículos que estos factores influyen en el grado de satisfacción de determinados ítems ${ }^{18,19,24,2627}$.

Por último, remarcar la importancia de realizar este tipo de estudios para conocer las expectativas de los pacientes, establecer puntos de mejora y mantener los puntos fuertes ya que los pacientes nos aportan opiniones y sugerencias muy relevantes para la mejora continua. Como se afirma en el estudio Factores asociados al estado de salud percibido (calidad de vida relacionada con la salud) de los pacientes en hemodiálisis crónica "Se ha demostrado que el nivel de satisfacción de los pacientes con la atención sanitaria que reciben se correlaciona con un índice de calidad de vida relacionada con la salud y con su bienestar psicológico"132.

Las limitaciones de este estudio son las inherentes a los diseños cualitativos debido a las pequeñas muestras utilizadas. No se pueden inferir los resultados al resto de la población.

\section{Consideraciones prácticas}

Desde el punto de vista práctico, es importante remarcar la importancia de realizar este tipo de estudios para conocer las expectativas de los pacientes, establecer puntos de mejora y mantener los puntos fuertes ya que los pacientes aportan opiniones y sugerencias muy relevantes para la mejora continua en la asistencia sanitaria. También es importante que los profesionales conozcan esta información porque debe suponer un aliciente en su trabajo y como es lógico el punto de partida para poder satisfacer las necesidades del paciente en hemodiálisis, que por ser un tratamiento crónico mantiene de por vida a algunos pacientes en el mismo. 


\section{Conclusiones}

De forma general los pacientes se muestran muy satisfechos con la atención recibida por parte de las enfermeras de la Unidad de Diálisis, destacando la seguridad que sienten cuando son atendidos en esta Unidad.

Asimismo, destaca de forma general, la positiva percepción del buen trato que reciben de las enfermeras, expresado en términos de "humano", "cercano" y "amable", apuntando que el interés que éstas profesionales demuestran hacia ellos, va más allá del interés propio por su situación de salud.

También tienen un alto grado de confianza en el personal de enfermería, basado en su destreza para pinchar la fístula arteriovenosa y en su fiabilidad profesional, muy por encima de otras competencias profesionales.

Recibido: 15 agosto 2016

Revisado: 19 agosto 2016

Modificado: 23 agosto 2016

Aceptado: 12 octubre 2016

\section{Bibliografía}

1. Bautista R Luz Marina. Percepción de la calidad del cuidado de enfermería en la ESE Francisco de Paula Santander. Aquichán [Internet]. 2008. 8( 1 ): 74-84. Disponible en: http://www. scielo.org.co/scielo.php?script=sci_arttext\&pi$d=S 165759972008000100007 \& \operatorname{lng}=e n$ (Consultado 9 Mayo 2016).

2. Cueto, E. G., Boubeta, A. R., \& Mallou, J. V. Presentación de una escala de satisfacción con los servicios sanitarios de atención primaria. Psicothema, [Internet] 2003; 15(4), 656-661. Disponible en: http://hdl.handle.net/10651/26748 (Consultado 9 Mayo 2016).

3. Mira, J. J., Buil, J. A., Aranaz, J., Vitaller, J., Lorenzo, S., Ignacio, E.,.. \& Giménez, A. ¿Qué opinan los pacientes de los hospitales públicos? Análisis de los niveles de calidad percibida en cinco hospitales. Gaceta Sanitaria. [Internet] 2003,14(4), 291293. Doi:10.1016/S0213-9111(00)71479-8.

4. Mira JJ, Aranaz J. La satisfacción del paciente como una medida del resultado de la atención sanitaria. Med Clin (Barc). [Internet] 2000;114 Supl 3:26-33. Disponible en: http://caliterevista. umh.es/indep/web/satisf_paciente.pdf (Consultado 9 Mayo 2016). 5. Car-Hill RA. The measurement of patient satisfaction. J Public Health. 1992; 14:236-49.

6. Donabedian A. Evaluating the quality of medical care. Milbank Memorial Fund Quarterly. 1966;44:166-202.

7. Monteagudo 0, Navarro C, Alonso P, Casas R, Rodríguez L, Gracia J; et al. Aplicación hospitalaria del SERVQHOS: facto- res asociados a la satisfacción e insatisfacción. Rev Calidad Asistencial. 2003;18:263-71.

8. Joseph C, Nichols S. Patient satisfaction and quality of life among persons attending chronic disease clinics in South Trinidad, West Indies. West Indian med. j. [serial on the Internet]. 2007; 56(2): 108-114. Available from: http://dx.doi. org/10.1590/S0043-31442007000200002. http:// docplayer.es/1577933-Mira-jj-aranaz-j-la-satisfaccion-del-pacientecomo-una-medida-del-resultado-de-la-atencion-sanitaria-medicina-clinica2000-114-supl-3-26-33.html (Consultado 9 Mayo 2016). 
9. Victoria García-Viniegras Carmen Regina, Rodríguez López Geidy. CALIDAD DE VIDA EN ENFERMOS CRÓNICOS. Revhabanciencméd [Internet]. 2007; 6(4). Disponible en: http:// scielo.sld.cu/scielo.php?script=sci_arttext\&pi$d=S 1729519 \times 2007000400002 \& \operatorname{lng}=e s$. (Consultado 9 Mayo 2016).

10. Megivern K, Halm MA, Jones G. Measuring patient satisfaction as an outcome of nursing care. J NursCareQual. [Internet]. 1992;6:9-24. Disponible en: http://journals.Iww.com/jncqjournal/ Citation/1992/07000/Measuring_patien t_satisfaction_as_an_outcome_of.5.aspx (Consultado 9 Mayo 2016).

12. Chang $E$, Hacock $K$, Chenoweth $L$, Jeon $Y H$, Glasson J, Gradidge K, et al. The influence of demographic variables and ward type on elderly patients' perceptions of needs and satis- faction during acute hospitalization internacional. J NursPract. [Internet]. 2003;9:191-201. Doi: 10.1046/j.1440172X.2003.00420.x.

13. Schmidt L. A patients' perceptions of nursing care in the hospital setting. J AdvNurs. [Internet]. 2003; 44:393-9. Doi: 10.1046/j.03092402.2003. 02818.x.

14. Larrabee JH, Ostrow $C L$, Withrow $M L$, Janney MA, Hobbs GR, Burant C. Predictors of patient satisfaction with impatient hospital nursing care.Nurs Health.[Internet]. 2004; 27:254-8.Disponible en: doi: 10.1002/nur.20021.

15. Jafar A, Muayyad M. Patients' satisfaction with nursing care in Jordan. Int $\mathrm{J}$ Health Care Qual Assur [Internet]. 2003;16:279-85.Disponible en: http://dx.doi.org/10.1108/09526860310495660.

16. Wagner D, Bear M. Patient satisfaction with nursing care: a concept analysis within a nursing framework. J AdvNurs. [internet]. 2008;9:692701. Disponible en: doi: 10.1111/j.1365-2648.2008.04866.x.

17. Pulido Agüero, F., Arribas Cobo, P., \& Fernández Fuentes, A.Satisfacción de los pacientes en la consulta de enfermería de enfermedad renal crónica avanzada. Revista de la Sociedad Española de Enfermería Nefrológica. [Internet]. 2008;11(3), 167-172. Disponible en: http://scielo.isciii.es/scielo.php?script=sci_arttext\&pi$d=S 113913752008000300002 \&$ Ing=es. (Consultado 26 Mayo 2016).
18. Hernández Meca, M., Ochando García, A., Lorenzo Martínez, S., Orbes Cervantes, P., \& López Revuelta, K. Factores determinantes de la satisfacción del paciente en tratamiento renal sustitutivo. Revista de la Sociedad Española de Enfermería Nefrológica. [Internet]. 2008;10(1), 6-13. Disponible en: http://scielo.isciii.es/scielo.php?script=sci_arttext\&pid=S113913752007000100002\&Ing=es. (Consultado 26 Mayo 2016).

19. Anguera Guinovart, E., \& Martínez Bateman, F. Estudio multicéntrico sobre la calidad asistencial percibida por los pacientes en programa de hemodiálisis de Tarragona. Revista de la Sociedad Española de Enfermería Nefrológica.2008;14(3), 172-180. Disponible en: http:// scielo.isciii.es/scielo.php?script=sci_arttext\&pi$d=S 113913752011000300005 \&$ Ing=es. (Consultado 26 Mayo 2016).

20. Mira, J. J., Pérez-Jover, V., Lorenzo, S., Aranaz, J., \&Vitaller, J. La investigación cualitativa: Una alternativa también válida. Atención Primaria. [Internet]2004;34(4), 161-166. Disponible en: doi:10.1016/S02126567(04)78902-7 (Consultado 26 Mayo 2016).

21. Monteagudo 0, Navarro C, Alonso P, Casas R, Rodríguez L, Gracia J; et al. Aplicación hospitalaria del SERVQHOS: facto- res asociados a la satisfacción e insatisfacción. Rev Calidad Asistencial. [Internet]. 2003; 18:263-71. Disponible en: doi:10.1016/S1134-282X(03)77619-2.

22. Sociedad Española de Nefrología . [sede Web]. Valencia: senefro.org; [Internet] 2014. Disponible en: http://www.senefro.org/modules.php?name $=$ webstructure\&idwebstructure $=29$ (Consultado 10 Junio 2016).

23. Cerdá, J. M., Rodríguez, M. P., García, M. H., \& Gaspar, 0. S. Técnicas cualitativas para la investigación en salud pública y gestión de servicios de salud: algo más que otro tipo de técnicas. Gaceta Sanitaria. [Internet] 1999;13(4), 312-319. Disponible en: doi:10.1016/S0213-9111(99)713737.

24. Seguí Gomà, A., Amador Peris, P., \& Ramos Alcario, A. B.Calidad de vida en pacientes con insuficiencia renal crónica en tratamiento con diálisis. Revista de la Sociedad Española de Enfermería Nefrológica. [Internet] 2010; 13(3), 155-160. Disponible en: http://scielo.isciii.es/scielo.php?script=sci_arttext\&pid=S113913752010000300002\&Ing=es. (Consultado 10 Junio 2016). 
25. Miguel Montoya, M., Valdés Arias, C., Rábano Colino, M., Artos Montes, Y., Cabello Valle, P., Castro Prieto, N. D.,...\& Ortega Suárez, F. Variables asociadas a la satisfacción del paciente en una unidad de hemodiálisis. Revista de la Sociedad Española de Enfermería Nefrológica. [Internet] 2009; 12(1), 19-25. Disponible en: http:// scielo.isciii.es/scielo.php?script=sci_arttext\&pi$d=S 113913752009000100004 \& \operatorname{lng}=e s$. (Consultado 17 Junio 2016).

26. Pérez Domínguez Tais, Rodríguez Pérez Armando, Suárez Álamo Jesús, Rodríguez Castellano Leire, García Bello Miguel Ángel, Rodríguez Pérez José Carlos. Satisfacción del paciente en una Unidad de Diálisis: ¿Qué factores modulan la satisfacción del paciente en diálisis?.Enferm Nefrol [Internet]. 15( 2 ): 101-107. Disponible en: http://dx.doi. org/10.4321/S225428842012000200004. (Consultado 28 Junio 2016).

27. Miguel Montoya, M., Valdés Arias, C., Rábano Colino, M., Artos Montes, Y., Cabello Valle, P., Castro Prieto, N. D.,\& Ortega Suárez, F. Variables asociadas a la satisfacción del paciente en una unidad de hemodiálisis. Revista de la Sociedad Española de Enfermería Nefrológica. [Internet] 2009 12(1), 19-25. Disponible en: http:// scielo.isciii.es/scielo.php?script=sci_arttext\&pi$d=S 113913752009000100004 \& \operatorname{lng}=e s$. (Consultado 28 Junio 2016).

28. Hernández Meca $E$, Ochando García $A$, Lorenzo Martínez S, Más de Marco M I y Noria Anguiado M E. Satisfacción del paciente en diálisis y calidad de vida laboral de enfermería: marcadores de calidad. En: Comunicaciones presentadas al XXXI Congreso Nacional de la Sociedad Española de Enfermería Nefrológica. Madrid. Sociedad Española de Enfermería Nefrológica; 2006.p.253-260.
29. Prieto M.A., Escudero M.J., Suess A., March J.C., Ruiz A., Danet A. Análisis de calidad percibida y expectativas de pacientes en el proceso asistencial de diálisis. Anales Sis San Navarra [Internet]. 2011; 34(1): 21-31. Disponible en: http:// scielo.isciii.es/scielo.php?script=sci_arttext\&pi$d=S 113766272011000100003 \& \operatorname{lng}=e s$. (Consultado 28 Junio 2016).

30. Haas JS, Coook EF, Puopolo AL, Burstin HR, Cleary $P D, B r e n n a n T A$. Is the professional satisfaction of general internist associated with patient satisfaction? Journal of General Internal Medicine 2000; 15:122-128.

31. Landon BE, Reschovsky J, Blumenthal D. Changes in Career satisfaction among primary care and specialist physicians, 1997-2001. JAMA 2003; 289:442-449.

32. Álvarez-Ude, F. Factores asociados al estado de salud percibido (calidad de vida relacionada con la salud) de los pacientes en hemodiálisis crónica. Revista de la Sociedad Española de Enfermería Nefrológica. [Intenet]. 2001; 14, 64-68. Disponible en: http://revistaseden.org/files/art134_1.pdf (Consultado 28 Junio 2016). 